\title{
Towards the lowest-energy limit for light ions identification with silicon pixel-type detectors
}

\author{
N. Cieplicka-Oryńczak ${ }^{1,2, a}$, D. Mengoni ${ }^{3,4}$, M. Ciemała ${ }^{1}$, S. Leoni ${ }^{2,5}$, B. Fornal ${ }^{1}$, J.A. Dueñas ${ }^{6}$, S. Brambilla $^{2,5}$, \\ C. Boiano ${ }^{2,5}$, P.R. John ${ }^{3,4,7}$, D. Bazzacco ${ }^{3}$, G. Benzoni ${ }^{2}$, G. Bocchi ${ }^{2,5}$, S. Capra ${ }^{2,5}$, F.C.L. Crespi ${ }^{2,5}$, A. Goasduff ${ }^{8}$, \\ K. Hadyńska-Klęk ${ }^{8}$, E.W. Iskra ${ }^{1}$, G. Jaworski ${ }^{8}$, F. Recchia ${ }^{3,4}$, M. Siciliano ${ }^{4,8}$, D. Testov ${ }^{3,4}$, and J.J. Valiente-Dobón ${ }^{8}$ \\ 1 Institute of Nuclear Physics Polish Academy of Sciences, PL-31342 Krakow, Poland \\ 2 Istituto Nazionale di Fisica Nucleare Sezione di Milano, Via Celoria 16, I-20133 Milano, Italy \\ 3 Istituto Nazionale di Fisica Nucleare Sezione di Padova, I-35131 Padova, Italy \\ 4 Dipartimento di Fisica dell'Università degli Studi di Padova, I-35131 Padova, Italy \\ 5 Università degli Studi di Milano, Via Celoria 16, I-20133 Milano, Italy \\ ${ }^{6}$ Departmento de Ingeniería Eléctrica y Centro de Estudios Avanzados en Física, Matemáticas y Computación, Universidad \\ de Huelva, 21071 Huelva, Spain \\ 7 Institut für Kernphysik, Technische Universität Darmstadt, D-64289 Darmstadt, Germany \\ 8 Istituto Nazionale di Fisica Nucleare Laboratori Nazionali di Legnaro, I-35020 Legnaro, Italy
}

Received: 18 May 2018 / Revised: 28 September 2018

Published online: 3 December 2018

(c) The Author(s) 2018. This article is published with open access at Springerlink.com

Communicated by C. Ur

\begin{abstract}
An in-beam test of two pixel-type silicon detectors of the TRACE detector project has been performed at Laboratori Nazionali di Legnaro (Italy). The aim was to investigate the lowest kinetic energy values at which isotopic identification of heavy-ion reactions products with mass $A \sim 10$ is possible, by using a single-layer silicon detector. Two separate read-out chains, analog and digital, were used, and the Pulse Shape Analysis technique was employed to obtain the particle identification matrices for the digitally processed data. The results confirmed the high capability of the Pulse Shape Analysis method which can be used for light ion identification, with performances similar to the analog approach. Separation in both charge and mass was obtained for $\mathrm{Li}$ and Be isotopes, however, the presence of a significant background from alpha particles severely limited the data analysis in the lower energy region. Due to this effect, the identification of the light products $\left({ }^{7,6} \mathrm{Li}\right.$ isotopes) could be possible down to $\sim 24.5 \mathrm{MeV}$ only, while the ${ }^{9,7} \mathrm{Be}$ isotopes were separable down to $\sim 29 \mathrm{MeV}$. This gives the value of $<4 \mathrm{MeV} /$ nucleon as the lowest kinetic energy for light products identification by using the pixel-type detectors of the TRACE project, in the present experimental conditions.
\end{abstract}

\section{Introduction}

One of the modern detection methods, offering identification of the reaction products with very low energy, is the Pulse Shape Analysis (PSA) [1-4]. This method can be applied to the signals from silicon detectors allowing to identify particles stopped in a detector from the information delivered by that detector only. In the past, a number of experiments were performed aiming at testing the possibility of light ions separation with neutronTransmutation Doped (nTD) Si detectors. For example, in ref. [1], where a $300 \mu \mathrm{m}$ thick silicon detector with $200 \mathrm{~mm}^{2}$ active area was used to collect the species of accelerated ions, the $8 \mathrm{MeV} /$ nucleon identification limit has

\footnotetext{
${ }^{a}$ e-mail: natalia.cieplicka@ifj.edu.pl
}

been observed for carbon isotopes. In [2] the products populated by the $474 \mathrm{MeV}$ beam of ${ }^{32} \mathrm{~S}$ impinging on the $\mathrm{Al}$ target were studied by PSA applied on the signals from a $500 \mu \mathrm{m}$ thick Si detector with $400 \mathrm{~mm}^{2}$ active area. Controlled silicon detectors, i.e., with known resistivity uniformity, were used and the importance of this parameter (which should be $<1 \%$ ) for the ion identification by nTD detectors was stressed. The authors quote $5 \mathrm{MeV} /$ nucleon (at $64 \mathrm{MeV}$ ) as the threshold for the isotopic identification of ${ }^{12} \mathrm{C}$ and ${ }^{13} \mathrm{C}$ nuclei. The identification of lighter nuclei, with $Z \leq 2$, was investigated using $500 \mu \mathrm{m}$ thick DoubleSided Silicon Strip Detector (DSSSD) [5]. The discrimination between $Z=1$ isotopes and between $Z=1$ and $Z=2$ nuclei was obtained down to energies of $2.5 \mathrm{MeV}$. Furthermore, in [6] the $500 \mu \mathrm{m}, 20 \times 20 \mathrm{~mm}^{2}$ nTD single pad Si detector, collimated $(\phi 3 \mathrm{~mm})$ to avoid the influence 
of the resistivity non-uniformity, was used for the tritiumdeuterium-proton identification produced in ${ }^{7} \mathrm{Li}+{ }^{12} \mathrm{C}$ reaction at $34 \mathrm{MeV}$. The obtained identification threshold for $Z=1$ and $Z=2$ was $\approx 3 \mathrm{MeV}$.

Measurements with the other type of Si detectors, that is, floating-zone (FZ) type, were also performed. As shown by Mengoni et al. [7], in the case of the TRACE array $[7,8]$, consisting of $200 \mu \mathrm{m}$ thick silicon modules and divided in 60 separately read pixels $\left(4 \times 4 \mathrm{~mm}^{2}\right.$ each $)$, the identification of the ${ }^{1,2,3} \mathrm{H}$ isotopes can be easily obtained down to energy of $4 \mathrm{MeV}$. In addition, the separation between ${ }^{3} \mathrm{He}$ and ${ }^{4} \mathrm{He}$ was also observed. This proved that the thin, FZ technique detector, with the uniformity guaranteed by the fine pad segmentation, may provide a good particle discrimination for reaction products heavier than ${ }^{3} \mathrm{He}$ and ${ }^{4} \mathrm{He}$, when the PSA technique is applied.

In this paper, we present the results of an in-beam experiment performed at the Legnaro National Laboratory, using segmented silicon detectors of the TRACE detector project $[7,8]$. The aim was to define the lowest kinetic energy at which reaction products, with mass $A \sim 10$, can be identified by Pulse Shape Analysis techniques. The ion-identification capability would be very important in a number of low-energy reaction studies, including, for example, nuclear structure investigations of light, neutronrich $\mathrm{Li}, \mathrm{Be}, \mathrm{B}, \mathrm{C}, \mathrm{N}, \mathrm{O}$ nuclei. These light nuclei are currently attracting a lot of interest, because they serve as the best testing ground for state-of-the-art theory approaches, which provide insight into the details of the nuclear force and the origin of nuclear clustering [9-12]. Particularly important in this context are the studies of the electromagnetic decay from unbound states, which is one of the most sensitive probes of the state wave functions. One of the possible ways to populate and characterize the decay of resonance states in light neutron-rich isotopes, is oneand two-nucleon transfer reactions induced by light-heavy ions on light-heavy targets, such as $\mathrm{Li}, \mathrm{Be}, \mathrm{B}, \mathrm{C}, \mathrm{N}$ and O. An example is the ${ }^{9} \mathrm{Be}\left({ }^{16} \mathrm{O},{ }^{14} \mathrm{O}\right){ }^{11} \mathrm{Be}$ reaction at approx. $14.5 \mathrm{MeV} /$ nucleon, used in ref. [13] to study excited states in ${ }^{11} \mathrm{Be}$ by detecting the binary partner ${ }^{14} \mathrm{O}$, with a high resolution magnetic spectrometer at forward angles. An alternative way would be to measure, directly, the target-like products: in this case they are ${ }^{11} \mathrm{Be}$ ions, scattered, with the highest cross section, mainly between $20^{\circ}$ to $40^{\circ}$, with energies from 6 to $15 \mathrm{MeV}$, which are rather low. Therefore, it becomes important to understand what is the lowest energy at which highly granular devices, like TRACE, would be capable of performing an isotopic identification of light-heavy ions. Our work shows a lower limit of approx. $3 \mathrm{MeV} /$ nucleon for target-like products of ${ }^{7,6} \mathrm{Li}$ and ${ }^{9,7} \mathrm{Be}$ in the present experimental conditions. This limit is higher than needed for the physics case discussed above, however we note that it could be probably improved by reducing the background from alpha particles, observed in the present experiment.

\section{Experimental set up and procedures}

The experiment was performed in July 2016 at the Legnaro National Laboratory (Italy). A ${ }^{37} \mathrm{Cl}$ beam, with en- ergy of $186 \mathrm{MeV}$ and intensity around $1 \mathrm{pnA}$ was focused on a $0.1 \mathrm{mg} / \mathrm{cm}^{2}$ thick ${ }^{12} \mathrm{C}$ target, in order to populate, with reasonable cross sections, the light-heavy nuclei of interest (e.g., Li, Be, B, C, .. ) , via a deep inelastic mechanism. Two TRACE silicon detectors were placed inside the reaction chamber. Each of them was made up of 60 $4 \times 4 \mathrm{~mm}^{2}$ pads with $4.5 \mathrm{~mm}$ pitch junction side, forming a matrix of $12 \times 5$ pads covering an area of approximately $50 \times 20 \mathrm{~mm}^{2}$. A common electrode covered the entire ohmic side of the detector. The ohmic side was a $260 \mathrm{~nm}$ thick As implanted layer without any metallization. The thickness of the detectors was $200 \mu \mathrm{m}$ and the resistivity of the float zone Silicon was about $15 \mathrm{kOhm} / \mathrm{cm}^{2}$. Due to the limited number of available read-out channels, only the signals from the common electrode and a few pads or groups of pads were recorded. The grouping of pixels was done on the PCB in the same way for both detectors: a group of 8 , two groups of 4 , two groups of 2 pads and three single pads were used per detector.

Both TRACE detectors were positioned at the forward angles, with the active area covering $\sim 35^{\circ}-57^{\circ}$, symmetrically with respect to the beam axis (where the maximum of the production of light ions was reached) and the ohmic side (the common electrode, denoted also as "BACK") facing the incoming particles. Such placement of the TRACE modules in the chamber, with the ohmic side facing the reaction products, was important to enhance the PSA capability. In this reverse mounting configuration a larger variety of signal shapes and larger charge collection times are obtained, as was shown in [4].

The detectors were connected with a $1 \mathrm{~m}$ long JUNKOSHA mini-coaxial cables (with a $0.5 \mathrm{~mm}$ diameter and a capacitance of $96 \mathrm{pF} / \mathrm{m}$ ) to a 16-channel chargesensitive preamplifier [14], designed at INFN Milano. The preamplifier gain was $45 \mathrm{mV} / \mathrm{MeV}$ yielding a dynamic range of approximately $100 \mathrm{MeV}$. The trigger of the acquisition was the signal from the common electrode. The signals from one detector were sent to the digital acquisition, while the second detector was connected to the analog read-out line. The voltage bias proceeded via a dedicated line, which is different from that of the signal readout, and it was uniformly applied to all the detector. The bias used during the experiment was $45 \mathrm{~V}$ for digital and $38 \mathrm{~V}$ for analog read-out chain (with the nominal depletion voltage $<20 \mathrm{~V}$ ).

The chain of the digital acquisition (fig. 1(a)) allowed to collect the signals ("traces") digitized by the $100 \mathrm{MHz}$ 14 bit customized sampling board [15]. The recorded trace was $1 \mu \mathrm{s}$ long and consisted of 100 points taken with steps of $10 \mathrm{~ns}$ (fig. 2(a)). This length of the traces was chosen to assure recording the full rise time and at the same time minimize the amount of written data. The signals were recorded, digitized and afterwards processed to extract the relevant observables. The signals were collected both from the ohmic side of the detector as well as from a few groups of pads (or single pads).

The trigger of the TRACE acquisition, obtained in this case from a digital leading edge discriminator embedded in the module, was the signal from the common electrode 
(a)

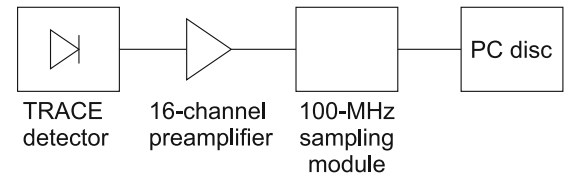

(b)

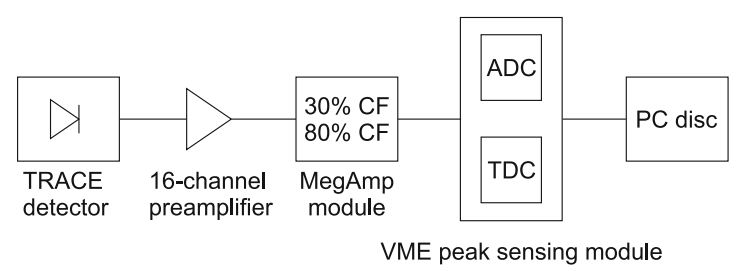

Fig. 1. The schemes of the acquisition lines for two TRACE detectors which were used during the experiment: (a) digital electronics, (b) analog electronics.

("BACK"). The signals from the pads were collected only if the BACK signal was present. The trigger rate of the signal coming from the group of 2 pads, for which the analysis was performed (see sect. 4), was $0.34 \mathrm{~Hz}$ with no dead time (100\% acquired).

The preamplifier signals from the second detector were processed by an analog chain (fig. 1(b)), using the MegAmp amplifier module [16] (also developed by INFN Milano), which provided energy and time information from a shaping amplifier (shaping time $0.5 \mu \mathrm{s}$ ) and two Constant Fraction Discriminators (CFD amplitude fractions $30 \%$ and $80 \%)$. Next, the signals were digitized by VME peak sensing ADC (V785 32 Channel Multievent Peak Sensing ADC) and TDC (V775 32 Channel Multievent TDC) CAEN modules. The trigger for the analog acquisition was constructed as the OR of the outputs of $30 \% \mathrm{CFD}$. The trigger rate of the signal coming from the group of 2 pads, as in case of the digital chain, was $0.34 \mathrm{~Hz}$, with dead time of $3 \%$.

\section{Data analysis}

In this section we present results of the analysis of digital and analog signals which was performed using the data analysis framework ROOT [17].

\subsection{Digital acquisition - Pulse Shape Analysis}

The analog charge signal from the output of the preamplifier was digitized by the $100 \mathrm{MHz}$ sampling module and processed offline. The baseline was calculated from 20 points $(200 \mathrm{~ns})$ and subtracted from the $1 \mu \mathrm{s}$ traces, after checking that no pile-up was present. The noise, i.e., 20 first points of the signal, showed, as expected, a Gaussian distribution with a FWHM which in most cases was less than $1 \%$ of the amplitude of the pulse (fig. 2(a)). In order to obtain the quantities allowing to separate detected reaction products, i.e., the maximum of the current signal $\left(I_{\max }\right)$ and the rise time $\left(T_{r}\right)$, the ROOT class TSpline3 [18] interpolation algorithm, ensuring the first

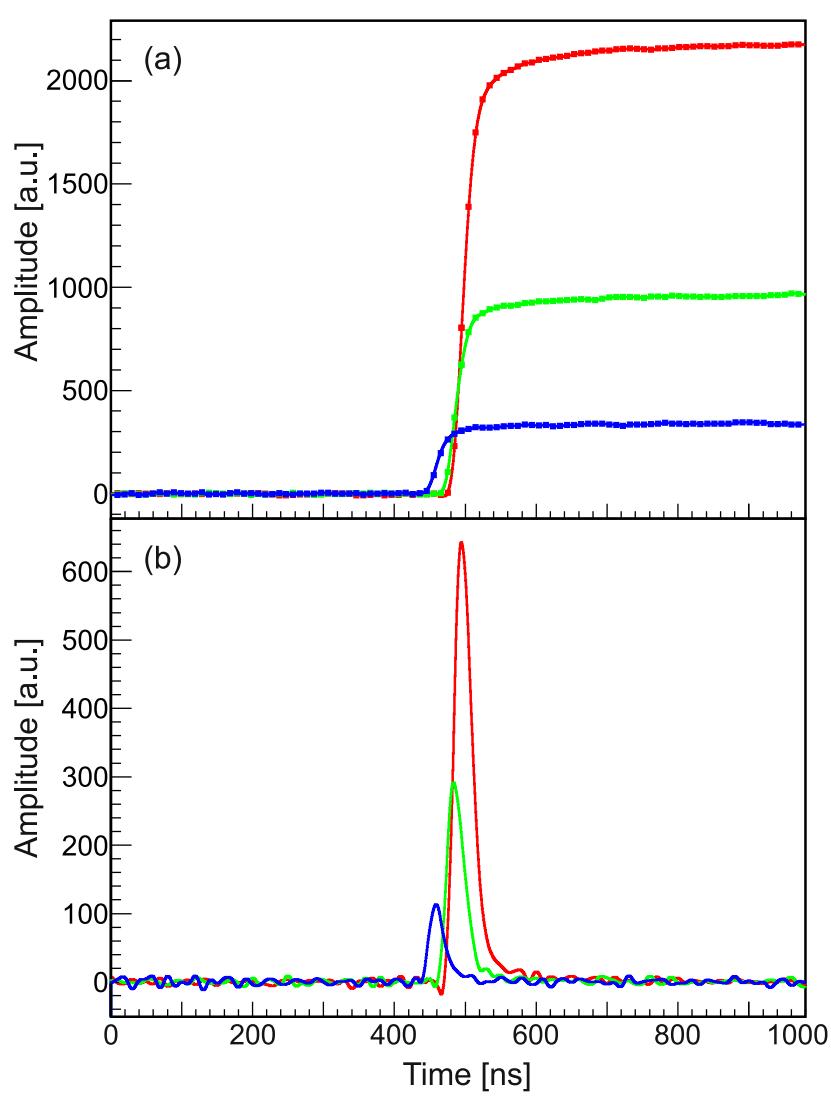

Fig. 2. The processing of the digital signal: (a) the examples of charge signal at the preamplifier output (the measured values are marked with points, while the solid lines are the interpolated curves) and (b) the derivatives of the interpolating function for each pulse, respectively.

and second derivative continuity, was applied. The precision of the calculation (10 ps) was chosen as a compromise between the computing time and sufficient precision. From the interpolated function, the rise time was obtained as leading edge discriminator, as $30-70 \%$ of the charge signal maximum which was calculated as the average of the 5 greatest values. The current signal was analytically extracted from the first derivative of the charge interpolated function. The 100 interpolated points, as showed in [6], were enough to calculate the stable maximum $\left(I_{\max }\right)$, see fig. 2(b).

The length of the recorded traces was $1 \mu \mathrm{s}$, which was sufficient to extract the $I_{\max }$ and $T_{r}$ values, however, to obtain the information on the energy, the longer traces were required. Therefore, the energy values were extracted from online (firmware) measurements. The online procedure allows for a longer shaping (integration) time which was optimized to $2 \mu \mathrm{s}$ for the silicon detectors - signals with a time window of $2 \mu$ s were processed in order to find the maximum proportional to the energy of the particle. The energy resolution was checked at the end of experiment using a three-peak alpha source $\left({ }^{239} \mathrm{Pu}^{2}{ }^{241} \mathrm{Am}-\right.$ ${ }^{244} \mathrm{Cm}$ with emission energies of $5.16,5.49$, and $5.80 \mathrm{MeV}$ ). The value of $30 \mathrm{keV}$ at $5.5 \mathrm{MeV}$ was obtained for the channel where only one pad was connected. However, due to 
the low statistics, the signal from a group of two pads was analyzed (see sect. 4). For this channel the energy resolution of $38 \mathrm{keV}$ was extracted. The values reported here and in sect. 3.2 include the impact of the energy lost in the dead layer.

As a first step towards the construction of the identification matrices, the energy correlation between the "BACK" (ohmic) and "PAD" (junction) sides was done. Only the events that generated the same energy on both sides of the detector $\left(E_{B A C K}=E_{P A D}\right.$, with tolerance in energy of $\Delta E \approx 3.4 \mathrm{MeV})$, i.e., the events lying on the diagonal of the $E_{B A C K}-E_{P A D}$ correlation matrix, were taken for further analysis. Fullfilling this condition assured that only the events when one pad (or group of pads) fired were considered.

The values of $I_{\max }$ and $T_{r}$, derived from the interpolated waveforms and correlated with the energy, allowed to construct the identification matrices and separate the light products (fig. 3(a) and (b)).

\subsection{Analog analysis}

The information from the analog acquisition of the second TRACE module provided the correlation between the energy of the particle and the time difference between the $80 \%$ and $30 \%$ of CFD (fig. 3(c)). Before constructing the identification matrix, the condition of $E_{B A C K}=E_{P A D}$ was applied, as previously done for the digital electronics. The energy resolution obtained using three-peak alpha source was $31 \mathrm{keV}$ at $5.5 \mathrm{MeV}$ both for a single pad and a group of two pads.

\section{Results}

The $186 \mathrm{MeV}$ beam of ${ }^{37} \mathrm{Cl}$ impinging on the ${ }^{12} \mathrm{C}$ target opened various direct and compound-nucleus reaction channels. In particular, light species, i.e., Li, Be, B isotopes, resulting from transfer reactions of a few nucleons, were produced at $\sim 40^{\circ}-60^{\circ}$, where the detectors were placed. On the contrary, the beam-like products could not be distinguished because they were out of the ADC dynamic range.

Although the best result in terms of the particle identification were obtained for electronic channels where only a single pad was connected (up to $15 \%$ of improvement comparing to the results presented here), due to limited statistics the analysis was performed considering two connected pads positioned at the average $\Theta$ angle $37^{\circ}$ with respect to the beam. We note that the other groups of pads, consisting of 4 or 8 pads, provided the best statistics, but the identification capability was not satisfactory in these cases. Representative examples of identification matrices presented in fig. 3(a), (b), and (c), correspond to 12 hours of measurement by the two connected pads.

The correlation matrix between the energy and the $I_{\max }$ constructed from the data recorded digitally by the first TRACE detector is presented in fig. 3(a). The energy scale is shown up to $\sim 76 \mathrm{MeV}$, while saturation was
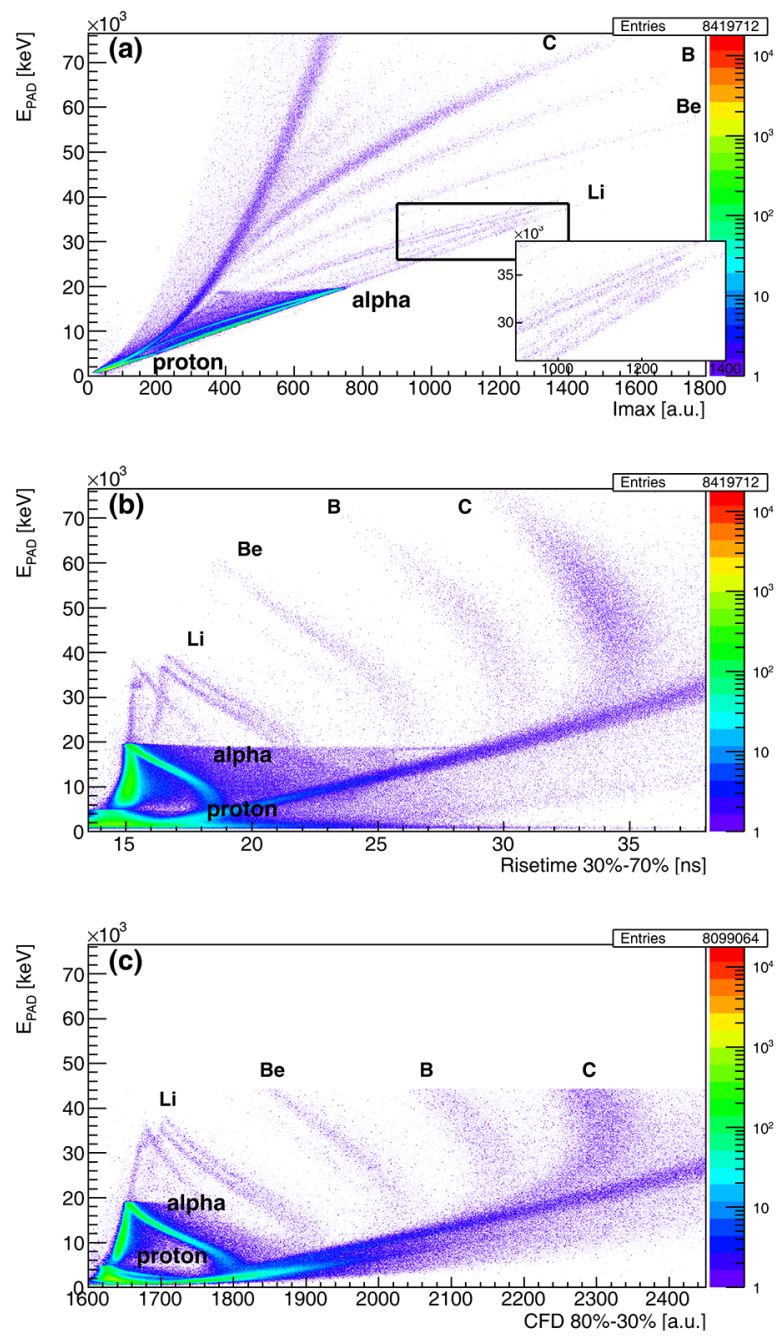

Fig. 3. Particle identification matrices constructed from the signal from a group of 2 PADs: (a) $E_{P A D} v s$. $I_{\max }$ (digital electronics), the inset shows the zoom of the region marked by a rectangle, (b) $E_{P A D}$ vs. $T_{r}$ (digital electronics), and (c) $E_{P A D}$ vs. 80-30\% CFD (analog electronics).

reached at around $93 \mathrm{MeV}$. The discrimination in $Z$ was easily achieved for protons, alpha particles, Li, Be, B, and $\mathrm{C}$ isotopes. The separation in $A$ is observed as well for the ${ }^{7,6} \mathrm{Li}$ and, with minor statistics, for ${ }^{9,7} \mathrm{Be}$. We note that the ${ }^{8} \mathrm{Be}$ nucleus is unbound and therefore cannot be seen in the matrix. This results in the easier identification of the ${ }^{9} \mathrm{Be}$ and ${ }^{7} \mathrm{Be}$ isotopes. The additional line in the Li region of the matrix, that reaches up to $\sim 1450 \mathrm{a}$.u. of $I_{\max }$ and up to $\sim 38 \mathrm{MeV}$ (inset of fig. 3(a)) was interpreted as coming from the events when two alpha particles were detected at the same time in the same pad giving the summed signal. This interpretation arises from the fact that the energy of $\sim 38 \mathrm{MeV}$ corresponds to the doubled value of the punch-through energy for alpha particles in case of the $200 \mu \mathrm{m}$ thick Si detector, i.e., $19.3 \mathrm{MeV}$.

The matrix of the energy vs rise time (30-70\%) correlation, extracted from the signal of the same TRACE module, is shown in fig. 3(b). Event distributions for alphas, 

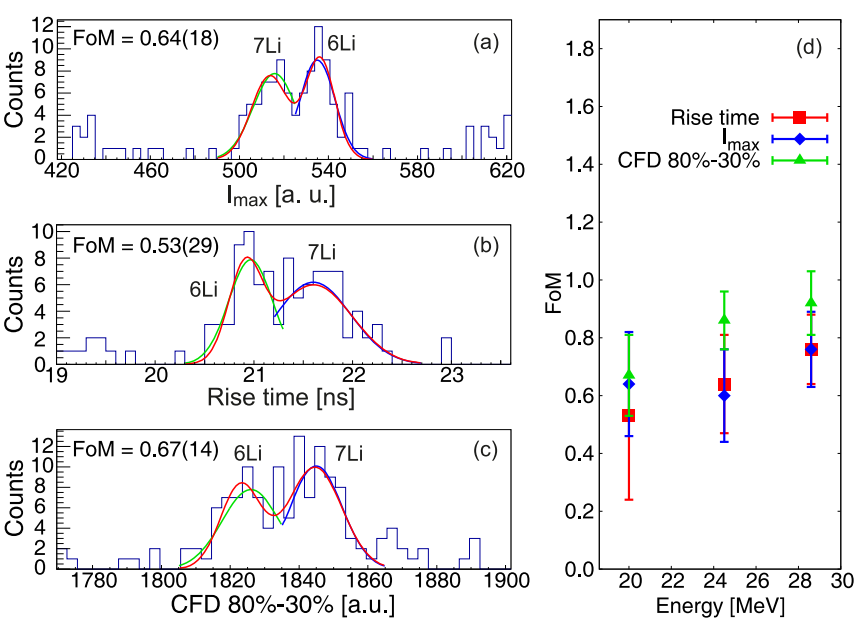

Fig. 4. Identification of ${ }^{7,6} \mathrm{Li}$ isotopes. Spectra for calculating the FoM obtained by projecting the $400 \mathrm{keV}$ wide cuts centered at $20 \mathrm{MeV}$ from the correlation matrices for 2 PADs: (a) $E_{P A D}$ vs. $I_{\max }$, (b) $E_{P A D}$ vs. $T_{r}$, and (c) $E_{P A D}$ vs. $80-30 \%$ CFD. The values of FoM for the three identification methods calculated for the energy ranges of 19.8-20.2, 24.3-24.7, and $28.4-28.8 \mathrm{MeV}$ are presented in panel (d) — see also table 1 .

Table 1. The values of $F_{O M}$ obtained with three different methods for the ${ }^{7,6} \mathrm{Li}$ isotopes are presented in columns $2-4$. The $400 \mathrm{keV}$ wide energy cuts were centered at the values given in column 1 -see also fig. 4 for graphical illustration.

\begin{tabular}{|c|c|c|c|}
\hline$E_{P A D}[\mathrm{MeV}]$ & $F o M I_{\max }$ & FoM $T_{r}$ & FoM $80-30 \%$ CFD \\
\hline 20.0 & $0.64(18)$ & $0.53(29)$ & $0.67(14)$ \\
\hline 24.5 & $0.60(16)$ & $0.64(17)$ & $0.86(10)$ \\
\hline 28.6 & $0.76(13)$ & $0.76(14)$ & $0.92(11)$ \\
\hline
\end{tabular}

${ }^{7,6} \mathrm{Li}$ and ${ }^{9,7} \mathrm{Be}$ are again clearly separated, and events coming from two-alpha particles, simultaneously detected, are also observed on the left side of the Li distribution.

In both matrices of fig. 3(a) and (b) one can notice a significant background which covers the range below $20 \mathrm{MeV}$. Most probably it was caused by alpha particles, as the upper energy limit of this background coincides with the punch-through energy for alpha particles $(19.3 \mathrm{MeV})$. Such background strongly limited the capability of the products separation at the lowest energies and will be discussed in the next section.

In fig. 3(c) the results of the analysis of the data from the second TRACE detector, connected to the analog acquisition chain, are presented. In this case, energies up to $\sim 44 \mathrm{MeV}$ were recorded. The correlation matrix of the energy and the $80-30 \%$ CFD allows to distinguish between the protons, alpha particles, ${ }^{7,6} \mathrm{Li},{ }^{9,7} \mathrm{Be}, \mathrm{B}$, and $\mathrm{C}$ reaction products. The double-alpha events are seen in vicinity of the Li curve, as previously.

In order to quantify the mass separation capability using both digital and analog chains, a figure of merit (FoM) was calculated for the $\mathrm{Li}$ and Be isotopes, for which sufficient statistics was collected. The FoM value was defined
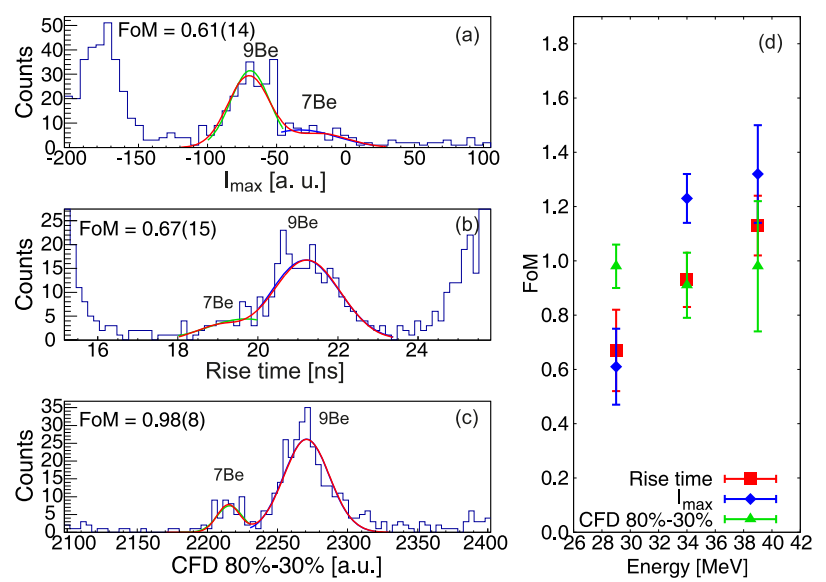

Fig. 5. Identification of ${ }^{9,7} \mathrm{Be}$ isotopes. Spectra for calculating the FoM obtained by projecting the $2 \mathrm{MeV}$ wide cuts centered at $29 \mathrm{MeV}$ from the correlation matrices for 2 PADs: (a) $E_{P A D}$ vs. $I_{\max }$, (b) $E_{P A D}$ vs. $T_{r}$, and (c) $E_{P A D}$ vs. $80-30 \%$ CFD. The cuts were rotated in order to project on the axis perpendicular to the identification curve. The values of FoM for the three identification methods calculated for the energy ranges of 28 $30,33-35$, and $38-40 \mathrm{MeV}$ are presented in panel (d) - see also table 2 .

Table 2. The values of FoM obtained with three different methods for the ${ }^{9,7} \mathrm{Be}$ isotopes are presented in columns $2-4$. The $2 \mathrm{MeV}$ wide energy cuts were centered at the values given in column 1 - see also fig. 5 for graphical illustration.

\begin{tabular}{|c|c|c|c|}
\hline$E_{P A D}[\mathrm{MeV}]$ & FoM $I_{\max }$ & FoM $T_{r}$ & FoM $80-30 \%$ CFD \\
\hline 29 & $0.61(14)$ & $0.67(15)$ & $0.98(8)$ \\
\hline 34 & $1.23(9)$ & $0.93(10)$ & $0.91(12)$ \\
\hline 39 & $1.32(18)$ & $1.13(11)$ & $0.98(24)$ \\
\hline
\end{tabular}

as in ref. [19], i.e.

$$
F o M=\frac{C_{2}-C_{1}}{F W H M_{1}+F W H M_{2}},
$$

where $C_{1}$ and $C_{2}$ are the positions of the centroids of the peaks corresponding to the ${ }^{7} \mathrm{Li}$ and ${ }^{6} \mathrm{Li}$ isotopes (fig. 4 and table 1 ) or ${ }^{9} \mathrm{Be}$ and ${ }^{7} \mathrm{Be}$ isotopes (fig. 5 and table 2 ). The errors of the extracted FoM values given in brackets (table 1 and table 2) were calculated based on the propagation of uncertainty from the standard deviations of $C_{1}$, $C_{2}, F W H M_{1}$, and $F W H M_{2}$.

Projections of the $400 \mathrm{keV}$ cuts centered at 20.0, 24.5, and $28.6 \mathrm{MeV}$ for $\mathrm{Li}$ isotopes and of the $2 \mathrm{MeV}$ cuts centered at 29, 34, $39 \mathrm{MeV}$ for Be isotopes, respectively, were done to obtain the FoM values from the identified peaks. Concerning Be isotopes, due to the wide energy windows used in the analysis, the cuts were projected on the axes perpendicular to the identification curves of interest, in order to reduce the energy spread. In this case, the use of wider energy windows was important to improve the statistics. 
The obtained values of $F o M$ for the ${ }^{7,6} \mathrm{Li}$ isotopes are reported in fig. 4 and table 1 for the different cuts and methods of identification. Figure 4(d) presents the dependence of the FoM values on the energy at which the cut was centered. It is found that the value $F o M=0.75$, usually considered as allowing for a good separation of the peaks [19], is achieved for the cut at $28.6 \mathrm{MeV}$ in the $E_{P A D}$ vs. $I_{\max }$ and $E_{P A D}$ vs. $T_{r}$ matrices (digital acquisition, $F O M=0.76$ for both methods) and for all the cuts starting from $20 \mathrm{MeV}$ and above in the $E_{P A D}$ vs. $80-30 \%$ CFD matrix (analog acquisition). However, one should note that there is an additional widening of the peaks caused by the large energy window taken to construct the spectra $\left(\Delta E_{P A D}=400 \mathrm{keV}\right)$, as the cuts were projected on the $\mathrm{X}$ axis in this case. Therefore, the results obtained even for $20 \mathrm{MeV}$, i.e., FoM values of $0.64(18)$ and $0.53(29)$ for $E_{P A D}$ vs. $I_{\text {max }}$ and $E_{P A D}$ vs. $T_{r}$, respectively, still allowing for identification of the ${ }^{7,6} \mathrm{Li}$ isotopes, can be considered as the low limit of energy for Li products identification in the present experimental conditions. We note that in the case of the $E_{P A D}$ vs. $80-30 \%$ CFD method, applied for the signal from the second TRACE detector, the identification was achieved also for the $400 \mathrm{keV}$ cut centered at $19.8 \mathrm{MeV}$ with $F o M=0.47(10)$.

The identification of ${ }^{9,7} \mathrm{Be}$ isotopes in terms of extracted FoM values was performed using $2 \mathrm{MeV}$ wide cuts, to compensate the minor statistics. The cuts were centered at 29,34 , and $39 \mathrm{MeV}$. The extracted FoM values for the three identification methods are reported in fig. 5(a)-(c) and table 2, while the plot $F o M\left(E_{P A D}\right)$ is presented in fig. $5(\mathrm{~d})$. For the lowest cut at $E_{P A D}=$ $29 \mathrm{MeV}, F O M$ values of $0.61(14)$ and $0.67(15)$ for the $E_{P A D}$ vs. $I_{\max }$ and $E_{P A D}$ vs. $T_{r}$ methods (digital acquisition) were obtained, respectively, showing a satisfactory identification ability, while the $F o M=0.98(8)$ value obtained for the analog $E_{P A D}$ vs. 80-30\% CFD method suggests that the lower limit of identification of Be isotopes has not been reached. However, projections at lower energies could not be used due to the very low number of counts. Additionally, the FoM values resulting from the use of $29-40 \mathrm{MeV}$ range of energy were calculated as: $0.93(4), 0.89(3)$, and $0.90(5)$ for the $E_{P A D}$ vs. $I_{\max }, E_{P A D}$ vs. $T_{r}$, and $E_{P A D}$ vs. 80-30\% CFD methods, respectively.

\section{Discussion}

The limits for low-energy light ions identification by PSA technique, obtained in the present experiment, are $\sim$ $20 \mathrm{MeV}$ for $\mathrm{Li}$ and $\sim 29 \mathrm{MeV}$ for Be isotopes. Concerning $\mathrm{Li}$ products, however, very good identification capability (i.e., FoM of $\sim 0.7$ in average) could be reached at $24.5 \mathrm{MeV}$ only. This value will then be taken as lowenergy limit for $\mathrm{Li}$ isotopes identification. We note that a further improvement could be achieved by reducing the background from alpha particles, which strongly limits the sensitivity around $20 \mathrm{MeV}$ in the present experimental conditions.
This background appears most probably due to the increased noise caused by radiation damage of the detector. We checked that in the matrices constructed from the data recorded during a short run at the beginning of the measurements this kind of background was not present. The question arose why in the analog processing of the signal this effect seems not to be so pronounced. We would interpret it as follows: the quantity used in the analog data analysis, that is the difference between $80 \%$ and $30 \%$ CFD, comes from the integration, thus, the noise is filtered. On the contrary, the method applied in PSA is by definition much more sensitive to the noise because it relies on the difference of the two interpolated values of the signal in case of $T_{r}$ or the derivative of the interpolated function $\left(I_{\max }\right)$. In this way, the larger noise at the end of the experiment affects significantly the extracted $T_{r}$ and $I_{\max }$. Moreover, the $100 \mathrm{MHz}$ sampling rate giving the measured signal at every $10 \mathrm{~ns}$ limits the precision of extracting $I_{\max }$ and $T_{r}$ which cannot be further improved by increasing the number of interpolated points.

The differences between the performances of the two acquisition systems used in the present experiment may come also from the fact that two separate signals from different detectors were processed. For example, the digitally read detector suffered from a leakage current of around $1.1 \mu \mathrm{A}$ at the end of the experiment $(0.2 \mu \mathrm{A}$ at the beginning), while a smaller value, $0.9 \mu \mathrm{A}(0.5 \mu \mathrm{A}$ at the beginning), was observed for the detector read with analog modules. Moreover, we cannot exclude that the ideal symmetry in position of both detectors with respect to the beam was not reached, which would result in slightly different level of radiation damage for the two TRACE detectors.

\section{Summary}

We presented the results of a test experiment aiming at defining the lowest kinetic energy values at which isotopic identification of heavy-ion reactions products with mass $A \sim 10$ is possible, by using two $200 \mu \mathrm{m}$ thick silicon TRACE detectors. Two acquisition systems, digital and analog, were employed to process the signals from the detectors. The digitized charge signal from one detector allowed to obtain the energy, maximum current $\left(I_{\max }\right)$, and rise time $\left(T_{r}\right)$ values, while the processing of the analog signal from the second detector provided energy and two time information signals from $30 \%$ and $80 \%$ Constant Fraction Discriminator (CFD). Three correlation matrices: energy vs. $I_{\max }, T_{r}$, and $80-30 \%$ CFD, representing three methods of identification, were constructed. All of them allowed for the separation of ${ }^{7,6} \mathrm{Li}$ and ${ }^{9,7} \mathrm{Be}$ isotopes in mass and charge. The present results confirmed the high quality of the Pulse Shape Analysis technique which was applied to the signals from Si detectors. This technique can be used for light ion identification as comparable with the analog approach. The calculated figures of merit for the cuts on different energies showed that the ability of isotope identification using energy vs. $I_{\max }$ and energy vs. $T_{r}$ 
methods is very similar within the uncertainties, for both ${ }^{7,6} \mathrm{Li}$ and ${ }^{9,7} \mathrm{Be}$ isotopes. Instead, the performance of the energy vs. 80-30\% CFD method, when considering the results for Li and Be products, was slightly better in the case of $\mathrm{Li}$ isotopes. One should stress that these two acquisition systems processed the signals from two separate TRACE modules, therefore the small differences in their performances may be caused by the different characteristics of these two detectors.

A good separation between the ${ }^{7,6} \mathrm{Li}$ isotopes was obtained down to the energy of $\sim 24.5 \mathrm{MeV}$, that is $\sim 3.5 \mathrm{MeV} /$ nucleon. Below this energy, a significant background coming from alpha particles appeared, severely limiting the identification capabilities. This might indicate that if one can improve the background reduction, a lower limit of particle identification can be reached. Owing to the absence of the bound ${ }^{8} \mathrm{Be}$ isotope, which enhanced the separation capability, the ${ }^{9,7} \mathrm{Be}$ isotopes were separable down to the energy of $\sim 29 \mathrm{MeV}$, that is $\sim 4 \mathrm{MeV} /$ nucleon. Also in this case, if the alpha background were significantly reduced, a lower energy limit could be achieved.

This work was supported by the Polish National Science Centre under Contract No. 2014/14/M/ST2/00738 (COPIN-INFN Collaboration).

Open Access This is an open access article distributed under the terms of the Creative Commons Attribution License (http://creativecommons.org/licenses/by/4.0), which permits unrestricted use, distribution, and reproduction in any medium, provided the original work is properly cited.

\section{References}

1. S. Barlini et al., Nucl. Instrum. Methods Phys. Res. A 600, 644 (2009).

2. L. Bardelli et al., Nucl. Instrum. Methods Phys. Res. A 654, 272 (2011).

3. S. Carboni et al., Nucl. Instrum. Methods Phys. Res. A 664, 251 (2012).

4. N. LeNeindre et al., Nucl. Instrum. Methods Phys. Res. A 701, 145 (2013).

5. M. Assié et al., Eur. Phys. J. A 51, 11 (2015).

6. J. Dueñas et al., Nucl. Instrum. Methods Phys. Res. A 676, 70 (2012).

7. D. Mengoni et al., Nucl. Instrum. Methods Phys. Res. A 764, 241 (2014).

8. D. Mengoni, PhD Thesis, Università di Camerino (2006).

9. H.G. Bohlen et al., Nucl. Phys. A 734, 345 (2004).

10. W. von Oertzen et al., Eur. Phys. J. A 21, 193 (2004).

11. W. von Oertzen, M. Freer, Y. Kanada-En'yo, Phys. Rep. 432, 43 (2006).

12. H.G. Bohlen et al., Eur. Phys. J. A 31, 279 (2007).

13. H.G. Bohlen et al., Nucl. Phys. A 722, 3c (2003).

14. E. Strano et al., Nucl. Instrum. Methods B 317, 657 (2013).

15. A. Pullia, D. Barrientos, D. Bazzacco, M. Bellato, D. Bortolato, R. Isocrate, in 2012 IEEE Nuclear Science Symposium and Medical Imaging Conference Record (NSS/MIC) (IEEE, 2012) p. 819.

16. C. Boiano, A. Guglielmetti, S. Riboldi, IEEE (NSS/MIC) Conference Record N14-34 (2012).

17. ROOT - An Object Oriented Data Analysis Framework, http://root.cern.ch/.

18. ROOT - An Object Oriented Data Analysis Framework, http://root.cern.ch/root/html/TSpline3.html.

19. J.A. Dueñas et al., Nucl. Instrum. Methods Phys. Res. A 714, 48 (2013). 